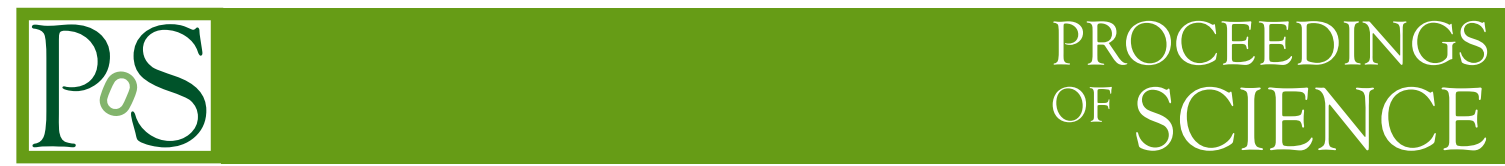

\title{
Numerical simulation of growing sandpiles
}

\section{Stefano Finzi Vita*}

Università di Roma La Sapienza

E-mail: finzi@mat.uniroma1.it

We discuss from a numerical point of view two recent mathematical models for growing sandpiles on a horizontal table under the action of a vertical source (see [3, 26] and [22]). Finite difference schemes for these models are proposed and analyzed in the one-dimensional case and then extended to the more realistic 2D case: they allow to compare the different dynamics and hence the different equilibria of the two models under general assumptions.

Control Systems: Theory, Numerics and Applications

30 March - 1 April 2005

Rome

${ }^{*}$ Speaker. 


\section{Introduction}

In the last ten years several papers have been devoted to the study of the dynamics of granular matter: many mathematical models have been derived using different techniques coming from kinetic, partial differential equations or cellular automata theories, see e.g. [3, 4, 5, 8, 9, 14, 15, 17, 22, 26, 27] and the references quoted therein.

Several years ago, Aronsson [2] suggested a system of ordinary differential equations to model the growth and the interaction of cones of sands produced by a point-wise source on an infinite table. It was only in [3] that such a model was reinterpreted in a rigorous way in the PDE settings: a more general variational model for growing sandpiles (in the sequel denoted by (VM)) was constructed as the limit of fast/slow diffusion evolution equations for the $p$-Laplace operator when $p$ goes to infinity. Independently, in a series of papers ([24, 25, 26]), Prigozhin reformulated the Dirichlet problem for a bounded table as an equivalent variational inequality, where the solution has to satisfy a constraint on the absolute value of the gradient (the maximal admissible slope, or critical slope, of the pile). It is worth saying that this formulation of the problem shows relevant connections with other interesting mathematical fields, such as the Monge-Kantorovich optimal mass transfer problem and the minimal Lipschitz extension problem (see for example [1, 16, 17, 18, 20] and [10, 23]).

According to the fundamental theories of de Gennes on granular matter (see [15]), more recently Hadeler and Kuttler [22] studied a system of two partial differential equations to model the growth of a sandpile under the action of a given vertical source. The main features of this model (denoted in the sequel as (HK)) is the distinction between two different layers in the pile, each of whom governed by a different law: a standing layer forming heaps and slopes and a small rolling layer running down the slope. A detailed characterization of the physically meaningful equilibrium solutions in dependence of the support of the source term was given, and explicit formulas were proved in one dimension and conjectured in two dimensions.

An interesting fact is that in principle, even if in the two previous approaches the dynamic is different, the set of equilibria is formally the same. It has been recently characterized in a rigorous mathematical setting by Cannarsa and Cardaliaguet [12] in two dimensions (and in any dimensions in [13]), making use of the theory of viscosity solutions.

Under suitable hypotheses on the support of the source function, the unique possible asymptotic equilibrium for both models is essentially given for the standing layer by the distance function from the boundary of the domain, which is also the maximal (viscosity) solution of the eikonal equation. In other cases, anyway, the steady-state is not uniquely determined a priori. For any time-independent source term, the solutions of $(\mathrm{VM})$ can be proved to evolve towards the minimal equilibrium configuration, characterized in terms of the maximum of all the critical cones centered at points of the support of the source term. In the (HK) model, on the contrary, positive rolling layers are allowed during the evolution even before the corresponding standing layer becomes critical. Then, the effective equilibrium configuration reached by the growing process has a larger support than the previous one, and it has not been yet mathematically characterized. In [19] we have shown through numerical experiments that the steady-state solutions depend in that case also on the source term intensity.

In this paper we want to study and compare the two models from a numerical point of view. 
In particular cases where explicit formulas for the steady-states are known, they can be directly computed, at least for simple geometries of the domain (fast algorithms to compute the distance function from the boundary of a set are known in the literature, see for example the fast marching method described in [11] and the references quoted therein). However, efficient methods for the solution of the evolutive PDE model are in general preferable, also for a complete description of the sandpile growth dynamics.

Here we propose a projected-diffusion finite difference scheme for the solution of (VM): in the first step the sand poured from the source is instantly diffused according to the sub-standing profile; in the second one we suitably project in order to keep subcritical the slope of the new profile. For the two-layer model (HK) we simply recall the explicit finite-difference approach introduced in [19] and its properties. We are then able to simulate the growth of sandpiles for several source terms according to the two different dynamical models, and to compare the corresponding steady-states in one and two dimensions.

The plan is the following: in Section 2 we describe the continuous models, whereas in Section 3 we introduce the numerical schemes and analyze their properties and efficiency in the onedimensional case. In the more interesting $2 \mathrm{D}$ case, the extensions are not always straightforward, and we discuss in Section 4 their implementation.

\section{The continuous models}

Let $\Omega$ be a bounded open domain of $\mathbf{R}^{2}$ (the table) and $Q_{T}=\Omega \times(0, T)$ the associated parabolic cylinder for a given time $T$. If $f$ is a vertical source pouring down sand on $\Omega$ as time goes by, we denote by $D_{f}$ the set of positivity of $f$ in $\Omega$ (so that $\bar{D}_{f}$ coincides with its support); a crucial role will be played by the function

$$
d(x):=\inf _{z \in \partial \Omega}|x-z|,
$$

which measures in any point the distance from the boundary of $\Omega$. The discontinuity set $\Re$ of $\nabla d$ is usually called the ridge set or the cut locus of $\Omega$. We review in this section two different dynamical models for growing sandpiles and we describe the set of their equilibria.

\subsection{A variational model and its highly nonlinear approximations}

A well-known model for sandpile growth has been studied a few years ago independently by Aronsson, Evans and Wu [3] and by Prigozhin [24, 25, 26]. In such a model the height $u$ of a pile growing under the effect of a nonnegative vertical source $f$ can be seen, by physical considerations, as the solution of the following problem:

$$
\begin{array}{cc}
u_{t}=\nabla \cdot(v \nabla u)+f & \text { in } \Omega \times(0, T) \\
|\nabla u| \leq 1, \quad|\nabla u|<1 \Rightarrow v=0 \quad \text { in } \Omega \times(0, T) \\
u=0 \quad \text { on } \partial \Omega, \quad u(\cdot, 0)=0 \quad \text { in } \Omega,
\end{array}
$$

where $v(x, t) \geq 0$ is an auxiliary function, still unknown, which plays the role of a dynamic Lagrange multiplier for the constraint on the gradient of $u$ (here we suppose for simplicity that the 
critical angle of repose of the pile is $\pi / 4$ ). Its positivity indicates that some matter, poured by the source, is rolling down along the surface of the pile which is critical in that point. The system (2.1)-(2.3) can be equivalently written as an evolutionary variational inequality

$$
u(t) \in K:\left(\partial_{t} u-f, \phi-u\right) \geq 0, \quad \forall \phi \in K, \forall t \in(0, T), \quad u(0)=0,
$$

on the convex set

$$
K:=\left\{\phi \in W_{0}^{1, \infty}(\Omega):|\nabla \phi| \leq 1\right\} .
$$

It was proved by penalization techniques that when $f \in L^{2}\left(Q_{T}\right)$ the problem (2.4) has a unique solution $u \in L^{\infty}\left(0, T ; W^{1, \infty}(\Omega)\right) \cap C^{1 / 4}\left(Q_{T}\right)$ such that $u_{t} \in L^{2}\left(Q_{T}\right)$. Moreover, $u$ is a non-decreasing function of time: since it is of course also bounded by the distance function $d$, then, if $f$ is independent of time, the solution $u(t)$ of (2.4) will evolve towards an equilibrium state $\bar{u} \in K$; we will discuss in Subsection 2.3 its characterization.

REMARK. Problem (2.4) can be written in a compact form as

$$
\begin{array}{cc}
f-u_{t} \in \partial I_{K}(u) & t>0 \\
u=0 & t=0,
\end{array}
$$

where $\partial I_{K}$ denotes the subdifferential of the indicator function of the convex set $K$. It can be interpreted as a mass transfer problem where the mass is instantaneously transferred from $f$ to the pile. For more details on Monge-Kantorovich optimal mass transfer theory in connection with sandpiles see e.g. [16, 17, 18, 20].

Another interesting way of deriving problem (2.4) is the following: let us consider the parabolic equations

$$
\begin{array}{r}
\partial_{t} u_{p}-\Delta_{p} u_{p}=f \quad \text { in } \Omega \times(0, T), \\
u_{p}=0 \quad \text { on } \partial \Omega \times(0, T), \quad u_{p}(0)=u^{0} \quad \text { in } \Omega,
\end{array}
$$

where

$$
\Delta_{p} w=\nabla \cdot\left(|\nabla w|^{p-2} \nabla w\right)
$$

is the known $p$-Laplacian operator, which for $p$ large may be regarded as a model operator for fast/slow diffusion processes; it was proved in [3] that, when $p \rightarrow \infty$, the solutions $u_{p}$ converge almost everywhere to a solution of (2.4); moreover, the solution of the very old ODE prototype model introduced by Aronsson [2] to describe the heights growing and the interaction of sand cones under the effect of point sources is the unique solution of (2.4). In other words, the dynamics of a growing sandpile can be approximated by the solution of the highly nonlinear problem (2.7)-(2.8) for $p$ large enough. In [7] a precise estimation of this approximation is proved:

$$
\left\|u-u_{p}\right\|_{L^{\infty}\left(0, T ; L^{2}(\Omega)\right)}^{2} \leq c\left[\frac{T}{p}+\inf _{v \in L^{2}(0, T ; K)}\left\|u_{p}-v\right\|_{L^{2}\left(0, T ; L^{2}(\Omega)\right)}\right],
$$

where $c$ is a positive constant independent from $p$.

We recall that in the corresponding stationary case (see [10] or [23]), the solutions of

$$
-\Delta_{p} u_{p}=f \quad \text { in } \Omega, \quad u_{p}=0 \quad \text { on } \partial \Omega
$$


for a nonnegative continuous function $f$ converge when $p$ goes to infinity to a function $u_{\infty}$ which solves, in the viscosity sense, the following equations

$$
\begin{array}{cl}
\left|\nabla u_{\infty}\right|=1 & \text { in } \quad D_{f} \\
\Delta_{\infty} u_{\infty} \equiv \frac{1}{2} \nabla u \cdot \nabla|\nabla u|^{2}=0 & \text { in } \quad \Omega \backslash \bar{D}_{f} .
\end{array}
$$

Then $u_{\infty}$ is the maximal solution of the first order eikonal equation in $D_{f}$, where it coincides with the distance function $d$, and solves a second order equation for the so-called infinity Laplacian operator $\Delta_{\infty}$ in the remaining part of $\Omega$.

\subsection{A two-layer dynamical system}

A different model for the dynamic of growing sandpiles was introduced in [22]. If we denote by $u$, respectively $v$, the heights of the standing and of the rolling layer of sand in the pile, that model consists in the following system of partial differential equations governing their evolution:

$$
\begin{array}{ccc}
v_{t}=\beta \nabla \cdot(v \nabla u)-\gamma(\alpha-|\nabla u|) v+f & \text { in } \Omega \times(0, T) \\
u_{t}=\gamma(\alpha-|\nabla u|) v & \text { in } \Omega \times(0, T) \\
u(\cdot, t)=0 \quad \text { on } \partial \Omega, \quad u(\cdot, 0)=0 \quad \text { in } \Omega, &
\end{array}
$$

which can be seen as the coupling of a transport equation for $v$ with a Hamilton-Jacobi type equation for $u$. The positive constants $\alpha, \beta$ and $\gamma$ denote, respectively, the critical angle of repose of the considered material, the characteristic diffusion and transport coefficients: again, for the sake of simplicity, and in view of a direct comparison with the solutions of model (2.4), we will assume in the sequel $\alpha=\beta=\gamma=1$.

No existence and uniqueness results for the solutions of an evolutive system such as (2.13)(2.15) are given in [22], but a careful analysis of the model shows its different dynamics. The standing layer $u$ stays all the time subcritical (that is, $|\nabla u| \leq 1$ ) as for the solution $u$ of (2.1)(2.3), but now the second strong condition in (2.2) only holds true for the steady-state: during the evolution a non-zero rolling layer $v$ can be active over a not yet critical $u$.

\subsection{Equilibria}

In both models the profile $u$ is monotone in time and bounded from above by the distance function $d$; then we expect $(u(t), v(t))$ to converge in time towards an equilibrium configuration $(\bar{u}, \bar{v})$ which has to solve in principle the same stationary system for the two cases:

$$
\begin{array}{cc}
-\nabla \cdot(v \nabla u)=f & \text { in } \Omega \\
(1-|\nabla u|) v=0 & \text { in } \Omega \\
\left.u\right|_{\partial \Omega}=0 . &
\end{array}
$$

We deduce that $D_{f} \subset\{\bar{v}>0\}$, and that $\bar{u}$ is not in general uniquely determined in regions where $\bar{v}=0$ (the previous system does not give any information on $\bar{u}$ in that case). Then it is interesting to characterize the real equilibria for the two models. A complete and rigorous description of solutions of (2.16)-(2.18) in two dimensions has been recently proved by Cannarsa and Cardaliaguet (see [12]), making use of the theory of viscosity solutions: 
Theorem 1. Let $\Omega \subset R^{2}$ be a bounded domain with $C^{2}$ boundary and $f \geq 0$ a continuous function in $\Omega$. Then there exists a solution of system (2.16)-(2.18) given by the pair $(u, v)$, where $u=d$ in $\Omega, v=0$ in $\Re$ and

$$
v(x)=\int_{0}^{\tau(x)} f(x+t \nabla d(x)) \frac{1-(d(x)+t) k(x)}{1-d(x) k(x)} d t, \quad \forall x \in \Omega \backslash \bar{\Re} .
$$

Such a solution is unique in the sense that if $\left(u^{\prime}, v^{\prime}\right)$ is another solution, then $v^{\prime}=v$ in $\Omega$ and $u^{\prime}=d$ in $\{x \in \Omega: v>0\}$.

In the previous formula, for any point $x \in \Omega, k(x)$ denotes the curvature of $\partial \Omega$ at the projection of $x$ onto it, while $\tau(x)$ indicates the so-called normal distance to $\bar{\Re}$, defined as

$$
\tau(x)=\min \{t \geq 0: x+t \nabla d(x) \in \bar{\Re}\} .
$$

Formula (2.19) says that $v$ is completely characterized, since it could be reconstructed at any point by integrating the source term along the shortest ray to the ridge set. The same does not hold true for $u$, which could be, where $v=0$, any subcritical function. According to the derivation of (VM) in terms of p-Laplacians, one could conjecture a role for $u_{\infty}$ at the equilibrium. When $D_{f}$ does not contain the whole ridge set $\Re$, it can be proved that the steady-state solution for (2.4) is

$$
u^{*}(x)=\max _{y \in D_{f}}(d(y)-|x-y|)^{+},
$$

which is the minimal one in the set of possible equilibria, and is not in general an infinity harmonic function in $\Omega \backslash \bar{D}_{f}$.

Even in [22] the unique physically meaningful equilibrium solution for the standing layer corresponding to a positive, constant in time, source term $f$, was stated to be the function $u^{*}$, but no proof was given for that. They only recall that the experiments on real sandpiles show in fact that as soon as the foot of the hill reaches the boundary in some point, all the sand starts rolling down through that point (the tip moves slightly in the opposite direction) so that the profile remains unchanged. In reality, the situation of the model (HK) looks essentially different: if the ridge set $\Re$ is contained in the set of positivity of $f$, then $u^{*}$ in (2.20) reduces to the distance function $d$ and it is of course the unique possible equilibrium. In any other situation, the uniqueness result of the previous theorem implies that $\bar{u}=u^{*}$ only in regions where $v$ differs from zero, i.e. at least inside the support of $f$; elsewhere the effective equilibrium $\bar{u}$ is only a subeikonal solution, that is a continuous nonnegative function such that $|\nabla u| \leq 1$ in $\Omega$, and it is in general always larger than $u^{*}$. This is a natural consequence of its different dynamics, and we clearly showed this result in [19] through some numerical experiments (see also next Sections 3 and 4). It still remains open, to our knowledge, a complete mathematical description of the effective equilibrium for this model and to which kind of granular matter the generally smoother profile of the growing piles is best-suited.

\section{The numerical study: one-dimensional case}

In the simple 1D case, we assume $\Omega=(0,1)$, so that $d(x)=\frac{1}{2}-\left|x-\frac{1}{2}\right|$ and the ridge set $\Re$ reduces to the middle point $x_{m}=0.5$. Then, in terms of equilibria, the theory implies that when 
$x_{m} \in \overline{D_{f}}$ the only possible steady-state is the maximal one, that is $\bar{u}=d$, for both the models. In any other case the two behaviors are different, as the experiments show. If $h=1 /(N-1)$ denotes the space discretization step, then over the interval $\Omega$ it is defined a uniform grid of nodes $x_{i}=(i-1) h$, for $i=1, . ., N$. The time discretization step will be denoted by $\Delta t$, the discrete times by $t_{n}=n \Delta t$, and $u_{i}^{n}$ will indicate an approximate value for $u\left(x_{i}, t_{n}\right)$.

\subsection{A variational approach via the $p$-Laplacians}

The possible derivation of problem (2.4) via the $p$-Laplacians already seen in Section 2.1 suggests, among the possible numerical approaches, the direct discretization of equation (2.7) for a large $p$. This way is highly inefficient, due to the strong nonlinearity of the problem, and we reported it only for the sake of comparison. A complete study for the finite element approximation of a parabolic $p$-Laplacian equations like (2.7) can be found for example in [ø], where, using $P_{1}$ finite elements, it was proved that

$$
\left|u_{p}(\cdot, n \Delta t)-u_{p}^{h}(\cdot, n \Delta t)\right|_{L^{2}(\Omega)} \leq c_{p}\left[h+(\Delta t)^{1 / 2}\right],
$$

which implies an $O(h)$ error estimate for $p$ fixed and $\Delta t=h^{2}$. Combining estimates (2.9) and (3.1) with the same choice for $\Delta t$ formally yields

$$
\left|u(\cdot, n \Delta t)-u_{p}^{h}(\cdot, n \Delta t)\right|_{L^{2}(\Omega)} \leq c\left(\sqrt{\frac{n}{p}}+c_{p}\right) h,
$$

which shows in particular that for $p$ fixed the accuracy gets lost as the number of time iterations increases. In practice this suggests, for a fixed $h$, to keep the quantity $n / p$ bounded: in other words, the parameter $p$ should increase with time. We tested the following $p$-growing numerical strategy:

- starting from a low $p_{0}$ solve (2.7) with linear finite elements in space and the backward Euler method in time;

- any fixed number of time iterations, replace $p_{i}$ by $p_{i+1}=p_{i}+\delta_{p}$, for a fixed, not too large, step $\delta_{p}$, using as initial datum for the new problem the last computed solution corresponding to $p_{i}$;

- repeat until stabilization.

This scheme is stable under the natural choice $\Delta t=h^{2}$, so that when the number of nodes grows, the evolution becomes very slow; another problem is that with this approach any particular sequence $\left\{p_{i}\right\}$ can strongly influence the final steady-state: the distance function $d$ correctly comes up whenever $x_{m} \in \overline{D_{f}}$, but in any other case the equilibrium state is in general a strictly convex function which is reminiscent of the contribution of the diffusion term corresponding to lower values of $p_{i}$ (see for example Figure 1).

\subsection{A discrete variational inequality}

In [24] two approaches were suggested for the direct numerical resolution of (2.4). In the first, a penalty method reduces the problem to a boundary value problem for the nonlinear parabolic equation

$$
\partial_{t} u_{\varepsilon}-\nabla \cdot\left(\left\{\frac{1}{\varepsilon}\left(\left|\nabla u_{\varepsilon}\right|^{2}-1\right)^{2}+\varepsilon\right\} \nabla u_{\varepsilon}\right)=f,
$$

which is then solved numerically. When $\varepsilon \rightarrow 0$ it can be proved that the solutions $u_{\varepsilon}$ converge to the solution $u$ of (2.4). 


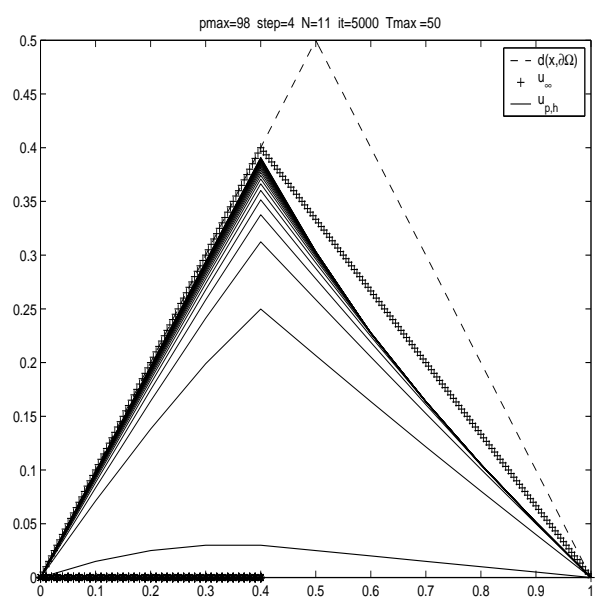

Figure 1: Limit of evolutionary $p$-Laplacian solutions when $D_{f}=(0,0.4)$ : the asymptotic solution when $p_{0}=2$, $p_{\max }=98$ and $\delta_{p}=4$, compared with $u_{\infty}(++)$ and $d(--)$.

Alternatively, an implicit semi-discretization in time may transform problem (2.4) into a sequence of stationary variational inequalities for each time step:

$$
u(t) \in K:(u(t)-(f \Delta t+u(t-\Delta t)), \phi-u) \geq 0, \quad \forall \phi \in K,
$$

which can be solved in space using piecewise linear finite elements. Since in that case the gradient of the approximated solution is constant inside every element, the problem (3.3) reduces to a constrained problem of convex programming, where augmented Lagrangian techniques can be applied.

Here we propose a different numerical scheme for the solution of problem (2.4), which uses the natural fact that the sand added to the pile in a particular point at any time may come either from the direct contribution of the source term $f$ over that point, or, by mass transport effects, from the one poured down to that point if there the slope is already critical. In order to keep the profile of the solution subeikonal, at any time step a projection procedure is applied. The algorithm for the one-dimensional problem is then the following:

$$
\begin{gathered}
u_{i}^{o}=0 \quad \forall i=1, \ldots, N ; \quad u_{1}^{n}=u_{N}^{n}=0 \quad \forall n ; \\
u_{i}^{n+1 / 2}=u_{i}^{n}+\Delta t \tilde{f}_{i}^{n} \quad i=2, \ldots, N-1 \\
u_{i}^{n+1}=P_{K_{i}^{n}}\left[u_{i}^{n+1 / 2}\right]
\end{gathered}
$$

where $\tilde{f}_{i}^{n}=f\left(x_{i}\right)$ if the slope is not critical in $x_{i}$ at time $t_{n}$; otherwise it represents the amount of sand poured down, instantaneously, along the critical slope from the neighboring nodes. For its evaluation a control variable $C$ is needed,

$$
C(i)=\left\{\begin{array}{cl}
0 & \left(\text { no transport in } x_{i}\right) \\
-1 & \text { (transport in left direction) } \\
+1 & \text { (transport in right direction) }
\end{array},\right.
$$

which is determined at each time step according to the values of the left and right derivative of the solution at any node. Moreover, for any time iteration and at any node of the grid

$$
K_{i}^{n}=\left\{v \in \mathbf{R}:\left|u_{i+1}^{n}-v\right| \leq h,\left|u_{i-1}^{n}-v\right| \leq h\right\},
$$


and

$$
P_{K_{i}^{n}}[v]=\max \left(\left(u_{i-1}^{n} \vee u_{i+1}^{n}\right)-h, \min \left(v,\left(u_{i-1}^{n} \wedge u_{i+1}^{n}\right)+h\right)\right)
$$

is the operator which projects onto $K_{i}^{n}$ (see Figure 2). Formula (3.6) is a standard way of implementing at a discrete level the gradient constraint for solutions of a variational inequality (see in [21] the problem of the elastic-plastic torsion of a bar). Since such operators $P_{K_{i}^{n}}$ are clearly

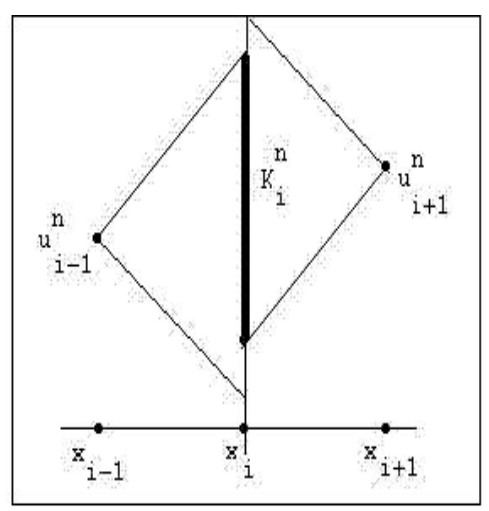

Figure 2: The set $K_{i}^{n}$.

monotone, the scheme produces a monotone increasing sequence $\left\{u^{n}\right\}$; in fact,

$$
u_{i}^{n} \leq u_{i}^{n+1 / 2} \Rightarrow u_{i}^{n}=P_{K_{i}^{n}}\left[u_{i}^{n}\right] \leq P_{K_{i}^{n}}\left[u_{i}^{n+1 / 2}\right]=u_{i}^{n+1}, \forall i
$$

when the middle point $x_{m}$ is in $\overline{D_{f}}$ such a sequence evolves very quickly (with a weak stability restriction $h=O(\Delta t)$ ) towards the profile of the distance function $d$; otherwise it can produce a reduced support pile or even a union of piles (see Figure 3), in accordance with the theory (formula (2.20) ) and with the physical experiments on real sandpiles. If we call $n_{i t}$ the number of necessary iterations to reach (up to a given tolerance) a discrete equilibrium, the stabilization time is given by $T_{\max }=n_{i t} \Delta t$. It is interesting to remark that when the equilibrium is the distance function, the convergence is faster and $n_{i t} \sim N$, so that $T_{\max }$ is essentially independent from $h$, as one would expect theoretically. In the other cases, $n_{i t} \sim N^{2}$ so that $T_{\max }$ linearly grows with $N$, which is of course unsatisfactory, and makes this approach inefficient for a large number of nodes (see Table 1). The reason is that in those cases the discrete evolution takes place at two different time scales: in the first one the pile rapidly grows under the direct action of the source, then the mass transport effects become dominant, and the pile keep on growing more slowly up to the equilibrium (see Figure 3 ). In the algorithm this second phase strongly depends on the number of nodes.

\subsection{A finite-difference scheme for the two-layer system}

Let us consider the one-dimensional version of (2.13)-(2.15) in $\Omega=(0,1)$, that is the system

$$
\begin{gathered}
v_{t}=v u_{x x}+v_{x} u_{x}-\left(1-\left|u_{x}\right|\right) v+f \quad \text { in } \Omega \times(0, T) \\
u_{t}=\left(1-\left|u_{x}\right|\right) v \quad \text { in } \Omega \times(0, T) \\
u(0, t)=u(1, t)=0, \quad u(x, 0)=0 \quad x \in \Omega .
\end{gathered}
$$




\begin{tabular}{|r||r|c|c||r|r|r|}
\hline \multicolumn{1}{||||}{} & \multicolumn{3}{c||}{$D_{f}=(0,1)$} & \multicolumn{3}{c|}{$D_{f}=(0,0.4)$} \\
\cline { 2 - 7 } $\mathrm{N}$ & $n_{i t}$ & $T_{\max }$ & $c p u-$ time & $n_{i t}$ & $T_{\max }$ & cpu-time \\
\hline \hline 11 & 11 & 1.1 & 0.016 & 21 & 2.1 & 0.016 \\
21 & 21 & 1.05 & 0.016 & 73 & 3.65 & 0.078 \\
51 & 51 & 1.02 & 0.078 & 421 & 8.42 & 0.609 \\
101 & 101 & 1.01 & 0.203 & 1641 & 16.4 & 3.782 \\
\hline
\end{tabular}

Table 1: Number of iterations, stabilization and cpu-times of the projected diffusion algorithm for different source supports.
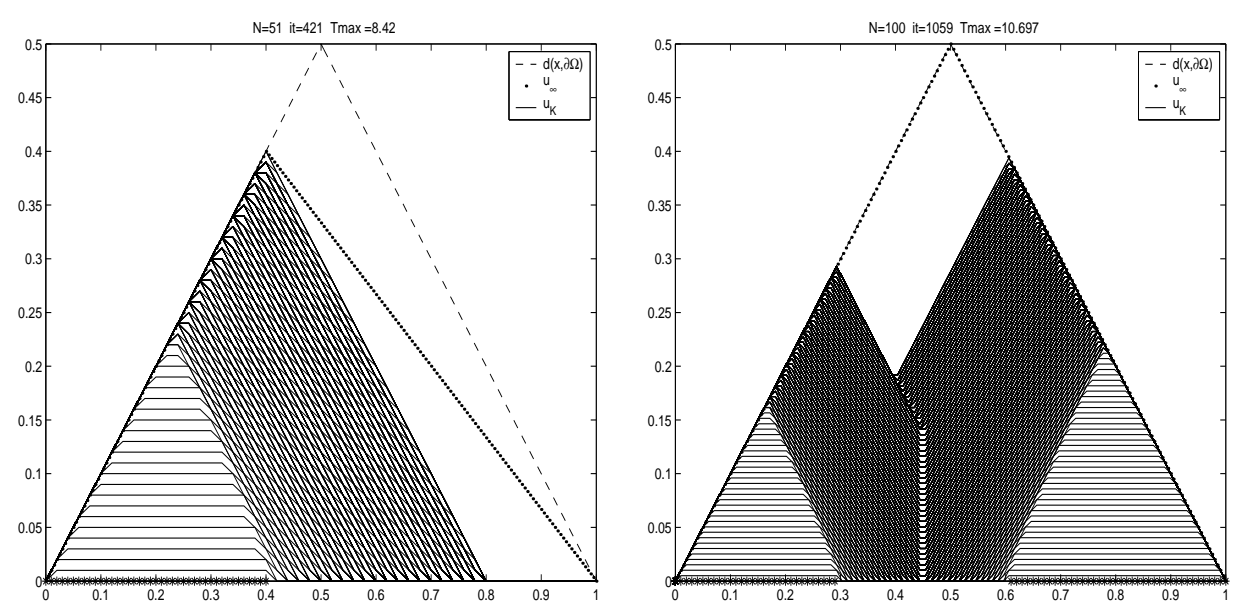

Figure 3: Growing sandpiles via the projected diffusion algorithm: a) $D_{f}=(0,0.4)$, a reduced support steady-state; b) $D_{f}=(0,0.3) \cap(0.6,1)$, a union of two piles.

At any node $x_{i}$ we set by definition

$$
D^{+} u_{i}=\frac{u_{i+1}-u_{i}}{h}, \quad D^{-} u_{i}=\frac{u_{i}-u_{i-1}}{h} .
$$

In [19] we discretized the derivative of $u$ at a node $x_{i}$ by the difference with maximal absolute value, namely

$$
D u_{i} \equiv \operatorname{maxmod}\left(D^{-} u_{i}, D^{+} u_{i}\right),
$$

whereas the corresponding derivative of $v$ was approximated by the upwind (with respect to $D u_{i}$ ) finite difference, i.e.

$$
\bar{D} v_{i} \equiv\left\{\begin{array}{cc}
D^{+} v_{i} & \text { if } D u_{i}>0, D^{+} u_{i}>0 \\
D^{-} v_{i} & \text { if } D u_{i}<0, D^{-} u_{i}<0 \\
0 & \text { otherwise }
\end{array}\right.
$$

These choices are motivated by the different role of the derivatives $u_{x}$ and $v_{x}$ in the two equations. Since (3.7) is a pure advection equation in $v$, we adopted for $v_{x}$ the upwind strategy according to the sign of $u_{x}$. For the latter, we were interested at any node to identify the local characteristic slope, 
that is how much the standing layer is close to the critical one, in order to control its growing in equation (3.8): then, the best choice seems to be (3.11).

Using forward Euler for the time derivatives discretization, we finally obtained a fully explicit scheme for the solution of (3.7)-(3.9), that is, in standard notation,

$$
\begin{gathered}
v_{i}^{n+1}=v_{i}^{n}+\Delta t\left[v_{i}^{n} D^{2} u_{i}^{n}+\bar{D} v_{i}^{n} D u_{i}^{n}-\left(1-\left|D u_{i}^{n}\right|\right) v_{i}^{n}+f_{i}\right] \\
u_{i}^{n+1}=u_{i}^{n}+\Delta t\left(1-\left|D u_{i}^{n}\right|\right) v_{i}^{n} \quad \forall i, \forall n \\
u_{i}^{0}=v_{i}^{0}=0 \quad \forall i, \quad u_{1}^{n}=u_{N}^{n}=0 \quad \forall n
\end{gathered}
$$

where $D^{2} u_{i}^{n}$ denotes the usual central finite difference scheme for the second derivative $u_{x x}$. The above scheme preserves the physical characteristics of the continuous model, is consistent with it and the local error is $O(h)$ under a stability condition $\Delta t=O(h)$ (see [19]). The solutions $\left(u^{n}, v^{n}\right)$ converge for $n$ going to $+\infty$ to an equilibrium configuration $(\bar{u}, \bar{v})$ of the discrete system, satisfying $(1-|D \bar{u}|) \bar{v}=0$ : when $x_{m} \in \overline{D_{f}}$ then always $\bar{u}=d$, otherwise $\bar{u} \geq u^{*}$ is a subeikonal function which depends on the source term $f$ (see Figure 4).

REMARK. Recently we tested a slightly different scheme, which distinguishes between $u_{x}$ and $\left|u_{x}\right|$ in the finite difference discretization. The sign of $u_{x}$ determines in fact the transport direction for the rolling layer $v$ in (3.7): it is then reasonable to take for it at any node the direction of maximal ascent, that is

$$
D u_{i} \equiv\left\{\begin{array}{cc}
D^{+} u_{i} & \text { if } u_{i-1} \leq u_{i} \leq u_{i+1} \\
D^{-} u_{i} & \text { if } u_{i+1} \leq u_{i} \leq u_{i-1} \\
0 & \text { if } \max \left(u_{i-1}, u_{i+1}\right) \leq u_{i} \\
\operatorname{maxmod}\left(D^{-} u_{i}, D^{+} u_{i}\right) & \text { otherwise }
\end{array}\right.
$$

For $\left|u_{x}\right|$ we kept the previous maxmod strategy (3.11). This approach gives better results in the experiments, but qualitatively the steady-state solutions remain the same.

\section{The numerical study: two-dimensional extensions}

The schemes (3.4)- (3.6) and (3.12)-(3.14) can be naturally extended to the more realistic case of a two-dimensional table. It is in fact enough to consider the four cardinal directions, both for the sand transportation and the projection procedure in the first case, and for the maxmod and upwind definition of finite differences in the second one. We refer to [19] for more details about the latter algorithm. Here we analyze the first one in the simple case of a rectangular table $\Omega=(0,1) \times(0, b)$; if $h=1 /(N-1)$ is again the space discretization parameter, the corresponding uniform grid is now made by $N \times M$ nodes $x_{i, j}=((i-1) h,(j-1) h)$, for $i=1, \ldots, N$ and $j=1, \ldots, M$, being $M=[b / h]+1$. Since the boundary is not $C^{1}$, the ridge set $\mathfrak{R}$, which is in this case a union of segments, touches the boundary at the four vertices of the table. It can be proved that formula (2.19) still holds in this case, because $\Re$ subdivides $\Omega$ in distinct regions and $\partial \Omega$ is piece-wise $C^{2}$. The projected-diffusion scheme (which will be denoted in the sequel $A \lg _{4 / 4}$ ) then becomes

$$
\begin{gathered}
u_{i, j}^{0}=0, \quad \forall i=1, . ., N, j=1, . ., M ; \\
u_{1, j}^{n}=u_{N, j}^{n}=0 \quad \forall j=1, . ., M ; \quad u_{i, 1}^{n}=u_{i, M}^{n}=0 \quad \forall i=1, . ., N, \forall n ; \\
u_{i, j}^{n+1 / 2}=u_{i, j}^{n}+\Delta t \tilde{f}_{i, j}^{n}, \quad i=2, . ., N-1, j=2, . ., M-1 ;
\end{gathered}
$$



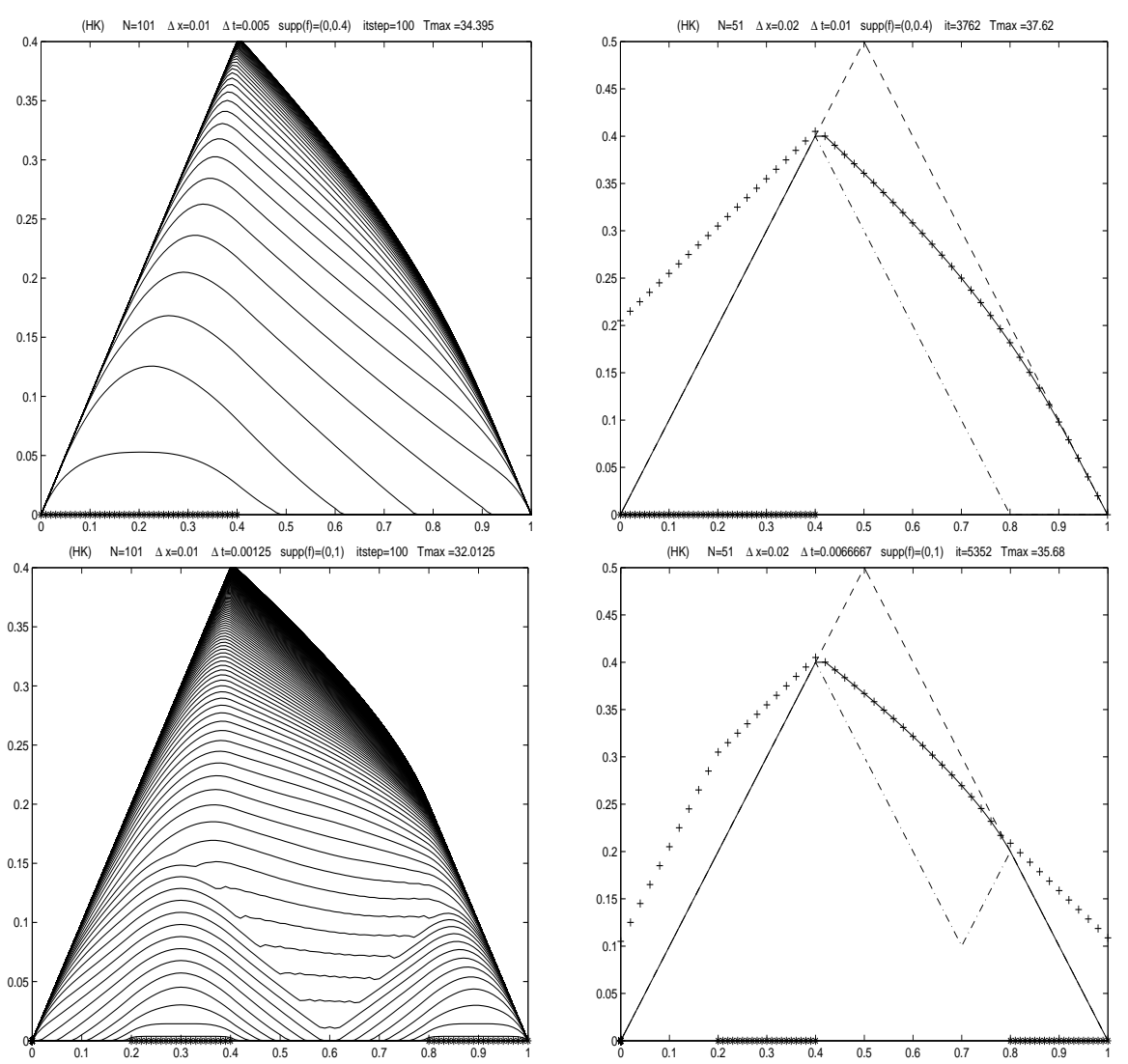

Figure 4: Growing sandpiles via the two-layer algorithm for a constant source (left); comparison of the steady-states $\bar{u}$ $[-]$ and $(\bar{u}+\bar{v})[++]$ with the the distance function $d[--]$ and the minimal solution $u^{*}[-\cdot-]$ (right); $D_{f}=[0,0.4]$ (top), $D_{f}=[0.2,0.4] \cup[0.8,1]$ (bottom).

$$
u_{i, j}^{n+1}=P_{K_{i, j}^{n}}\left[u_{i, j}^{n+1 / 2}\right]
$$

where $\tilde{f}_{i, j}^{n}$ is the corrected source term at time $t_{n}$, which coincides with $f\left(x_{i, j}\right)$ only if in none of the four orthogonal directions the slope is critical. Otherwise, $f\left(x_{i, j}\right)$ is equally distributed to the adjacent nodes at critical slope with respect to $x_{i, j}$. This procedure is repeated until there is no more sand to transport. In natural notations,

$$
K_{i, j}^{n}=\left\{v \in \mathbf{R}^{2}:\left|u_{i+1, j}^{n}-v\right| \leq h,\left|u_{i-1, j}^{n}-v\right| \leq h,\left|u_{i, j+1}^{n}-v\right| \leq h,\left|u_{i, j-1}^{n}-v\right| \leq h\right\},
$$

and the four-directions projection operator is given by

$$
\begin{gathered}
P_{K_{i, j}^{n}}[v]=\max \left[\max \left(\left(u_{i-1, j}^{n} \vee u_{i+1, j}^{n}\right)-h, \min \left(v,\left(u_{i-1, j}^{n} \wedge u_{i+1, j}^{n}\right)+h\right)\right),\right. \\
\left.\max \left(\left(u_{i, j-1}^{n} \vee u_{i, j+1}^{n}\right)-h, \min \left(v,\left(u_{i, j-1}^{n} \wedge u_{i, j+1}^{n}\right)+h\right)\right)\right] .
\end{gathered}
$$

If $\Re \subset \overline{D_{f}}$ we already know from the theory that the solution evolves towards the distance function $d$, which is of course a squared pyramid when $\Omega$ is a square or a "tent-shaped" pile when it is a rectangle. In these cases the algorithm behaves sufficiently well, and the final results are the same as for the (HK) model (see Figure 5). 

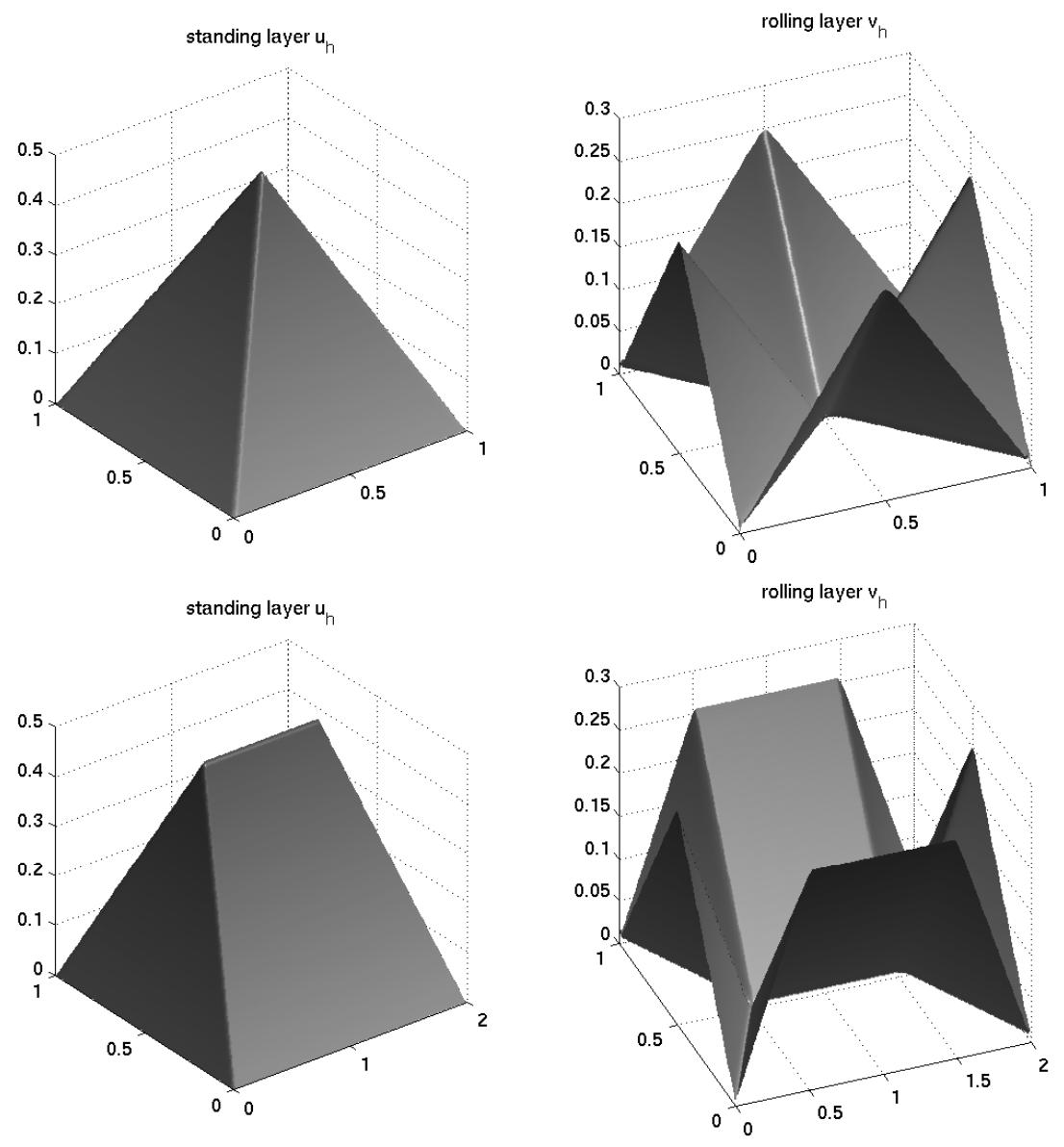

Figure 5: Maximal equilibria $u$ and $v$ when $D_{f}=\Omega ; \Omega=(0,1) \times(0,1)$ (top); $\Omega=(0,1) \times(0,2)$ (bottom).

In general, anyway, the use of a structured grid causes the sandpile to grow anisotropically, and to form pyramids (more than cones) oriented along the four main cross directions. Moreover, a supercritical slope could appear in some directions (see Figure 7 (center)). So when $D_{f}$ does not contain the whole ridge set the results are strongly unsatisfactory.

In order to overcome such problems, several attempts can be made. One idea could be simply to consider more directions for the sand transportation, for example adding the four diagonal directions, with a suitable correction for the corresponding adjacent nodes (which have now a distance $\sqrt{2} h$, and not $h$, from the node under consideration). Unfortunately, such a choice produces a larger expansion of the pile, which does not evolve towards the prescribed minimal solution $u^{*}$. The reason is that, as already mentioned, if the projection operator only controls four directions, the corresponding profile could be overcritical in any other direction, such as for example the diagonal ones. Then it is better to keep the transportation step as in formula (4.3), but to consider more directions for the projection step (4.4). The directions of the eight adjacent nodes could be enough, but more conical shapes are produced by a 16-directions projection applied on a double stencil which also includes the eight non-adjacent nodes at distance $\sqrt{5} h$ (see Figure 6 and 7 (bottom)). We called this new scheme $\mathrm{Alg}_{4 / 16}$ : in practice its projection procedure is now performed, for any 
strictly-internal node, in three subsequent steps:

$$
\begin{array}{cc}
u_{o r t}^{n+1}=P_{K}\left[u^{n+\frac{1}{2}}\right] & (4 \text { orthogonal directions }), \\
u_{o b l}^{n+1}=P_{K}\left[u_{o r t}^{n+1}\right] & (4 \text { diagonal directions }), \\
u^{n+1}=P_{K}\left[u_{o b l}^{n+1}\right] \quad(8 \text { intermediate directions) }
\end{array}
$$

In Table 2 we have tabulated the values of the $L^{\infty}$ norm of the error between the expected and the computed steady-state solutions for the two algorithms $\mathrm{Alg}_{4 / 4}$ and $\mathrm{Alg}_{4 / 16}$ with different choices of the source support. It is possible to see the great improvement gained by the second approach, even if it has to be remarked that it results very heavy in terms of computational time.

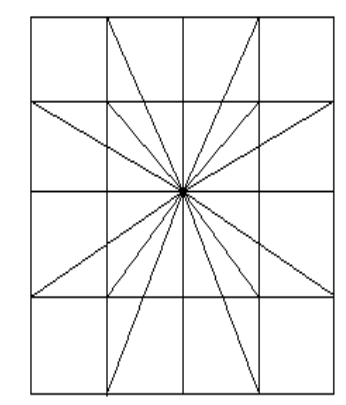

Figure 6: The 16-directions stencil used for the projection step in $A l g_{4 / 16}$.

\begin{tabular}{|c|c|c|c|}
\hline$D_{f}$ & $N$ & $A l g_{4 / 4}$ & $A l g_{4 / 16}$ \\
\hline$(0.4,0.6) \times(0.4,0.6)$ & 21 & 0.1103 & 0.0214 \\
& 41 & 0.1147 & 0.0162 \\
\hline$(0.6,1) \times(0.6,1)$ & 21 & 0.1137 & 0.0185 \\
& 41 & 0.1159 & 0.0147 \\
\hline$(0,1) \times(0,0.4)$ & 21 & 0.1047 & 0.0185 \\
& 41 & 0.1109 & 0.0147 \\
\hline$(0.4,0.6) \times(0.9,1)$ & 21 & 0.1410 & 0.0244 \\
& 41 & 0.1449 & 0.0185 \\
\hline
\end{tabular}

Table 2: $L^{\infty}$-norm of the error on the computed steady-state for different source supports.

\section{References}

[1] L. Ambrosio, Lecture notes on optimal transport problems, Summer School on 'Mathematical aspects of evolving interfaces', Madeira, preprint n. 32, Scuola Normale Superiore, Pisa, 2000

[2] G. Aronsson, A mathematical model in sand mechanics: presentation and analysis, SIAM J. Appl. Math., 22 (1972), 437-458

[3] G. Aronsson, L.C. Evans and Y. Wu, Fast/slow diffusion and growing sandpiles, J. Diff. Equations, 131 (1996), 304-335 

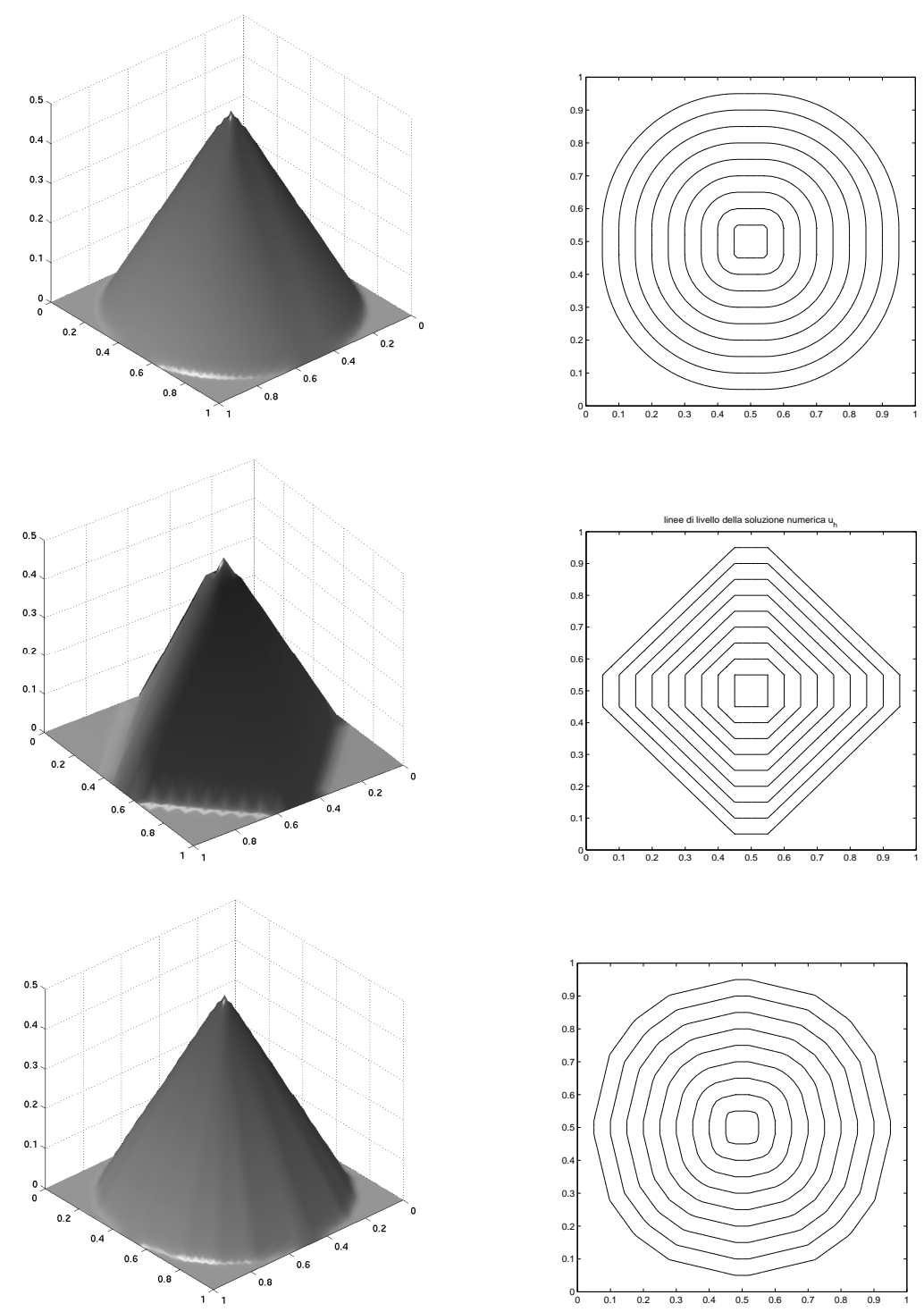

Figure 7: Growing sandpiles for the projected diffusion algorithm on the square table when $D_{f}=(0.45,0.55)^{2}, \mathrm{~N}=51$ : the expected steady-state solution and its level lines (top); the steady-state solutions computed with $\mathrm{Alg}_{4 / 4}$ (center) and $\mathrm{Alg}_{4 / 16}$ (bottom) and their level lines.

[4] P. Bak, How nature works. The science of self-organized criticality, Copernicus, New York, 1996

[5] P. Bak, Tang and Wiesenfeld, Self-organized criticality, Phys. Rev. A, 38 (1988), 364-374

[6] J.W. Barrett and W.B. Liu, Finite element approximation of the parabolic p-Laplacian, SIAM J. Numer. Anal., 31 (1994), 413-428

[7] J.W. Barrett and L. Prigozhin, Bean's critical-state model as the $p \rightarrow \infty$ limit of an evolutionary p-Laplacian equation, J. Nonlinear Anal., 42 (2000), 977-993

[8] R.P. Behringer, The dynamics of flowing sand, Nonlinear Sci. Today, 3 (1993), 1-15

[9] D. Benedetto, E. Caglioti and M. Pulvirenti, Collective behavior of 1-D granular media, in Modelling in Applied Science, A kinetic theory approach, Bellomo and Pulvirenti eds., Birkhauser (2000) 
[10] T. Bhattacharya, E. Di Benedetto and J. Manfredi, Limits as $p \rightarrow \infty$ of $\Delta_{p} u_{p}=f$ and related extremal problems, Rend. Sem. Mat. Univ. Pol. Torino, Special Issue (1989), 15-68

[11] E. Cristiani and M. Falcone, Fast semi-Lagrangian schemes for the eikonal equation and applications, Dipartimento di Matematica, Universitá di Roma "La Sapienza", preprint (2005)

[12] P. Cannarsa and P.Cardaliaguet, Representation of equilibrium solutions to the table problem for growing sandpiles, J. Eur. Math. Soc. (JEMS), 6 (2004), 435-464

[13] P. Cannarsa, P.Cardaliaguet, G. Crasta and E. Giorgieri, A Boundary Value Problem for a PDE Model in Mass Transfer Theory: Representation of Solutions and Applications, to appear in Calc. Var. Partial Differential Equations (2004)

[14] D. Dhar, Self-organized critical state of sandpile automation models, Phys. Review Letters, 64 (1990), 1613-1616

[15] P.G. de Gennes, Granular matter, Summer School on Complex Systems, Varenna, Lecture Notes Societá Italiana di Fisica (1996), 21-51

[16] L.C. Evans, Partial differential equations and Monge-Kantorovich mass transfer, Current Developments in Mathematics 1997, Int. Press, Boston, MA (1999), 65-126

[17] L.C. Evans, M. Feldman and R.F. Gariepy, Fast/slow diffusion and collapsing sandpiles, J. Diff. Equations, 137 (1997), 166-209

[18] L.C. Evans and W. Gangbo, Differential equations methods for the Monge-Kantorovich mass transfer problem, Memoirs of the A.M.S., 653 (1999)

[19] M. Falcone and S. Finzi Vita, A finite-difference approximation of a two-layer system for growing sandpiles, preprint (2005)

[20] W. Gangbo, The Monge mass transfer problem and its applications, Contemp. Math., 226 (1999), 79-104

[21] R. Glowinski, J.L. Lions and R. Trèmoliéres, Analyse numèrique des inèquations variationnelles, Dunod, Paris, 1976

[22] K.P. Hadeler and C. Kuttler, Dynamical models for granular matter, Granular Matter, 2 (1999), 9-18

[23] U. Janfalk, Behaviour in the limit, as $p \rightarrow \infty$, of minimizers of functionals involving p-Dirichlet integrals, SIAM J. Math. Anal., 27 (1996), 341-360

[24] L. Prigozhin, A variational problem of bulk solids mechanics and free-surface segregation, Chem. Eng. Sci., 78 (1993), 3647-3656

[25] L. Prigozhin, Sandpiles and river networks: extended systems with nonlocal interactions, Phys. Rev. E, 49 (1994), 1161-1167

[26] L. Prigozhin, Variational model of sandpile growth, Euro. J. Appl. Math., 7 (1996), 225-235

[27] H. Puhl, On the modelling of real sand piles, Phys. A, 182 (1992), 295-308 